\title{
The Monitoring of Immunosuppressive Therapy with Tacrolimus in Patients with Kidney Transplant, Based on the Pharmacokinetic Criteria
} \author{
Titica ${ }^{3}$, Carașca Emilian4, Dogaru Grigore Aloiziu ${ }^{1}$ \\ Department of Nephrology, University of Medicine and Pharmacy, Târgu-Mureș, România \\ 2 Department of Physiology, University of Medicine and Pharmacy, Târgu-Mureș, România \\ ${ }^{3}$ Department of Pharmacology and Clinical Pharmacy, University of Medicine and Pharmacy, Târgu-Mureș, România \\ ${ }^{4}$ Department of Internal Medicine IV, University of Medicine and Pharmacy, Târgu-Mureș, România
}

Stoica Ciprian Mihai ${ }^{1}$, Căldăraru Carmen Denise ${ }^{2}$, Vari Camil Eugen ${ }^{3}$, Tarța Dorin lonuț ${ }^{1}$, Dogaru Maria

Background: Therapeutic drug monitoring (TDM) in patients with Chronic Kidney Disease (CKD) with kidney transplant, represents a major post transplant concern due to the characteristics of this special category of patients, particularities which can generate changes of the pharmacokinetic profile of the administered medication.

Material and methods: The current study is a retrospective pharmacokinetic study, over a period of 50 months, including a group of 36 kidney transplanted patients with CKD. Tacrolimus blood concentration was determined by a validated high-performance liquid chromatography method (HPLC), at a 12 hour time interval from the last administration of the immunosuppressive medication and before the following dose (Residual concentration, Cmin(trough)).

Results: During the monitoring of therapy, based on the pharmacokinetic criteria, 252 measurements of blood concentration were determined, 58 of these being outside the therapeutic window.

Conclusions: The results obtained show that it is mandatory to continue to monitor closely medical therapy based on the pharmacokinetic criteria in view of improving drug administration. The other ways of monitoring therapy: the clinical and biochemical criteria should not be overlooked. In addition, the interindividual variability of patients should be considered, as well as drug interaction which can alter the pharmacokinetics of tacrolimus.

Keywords: chronic kidney disease, therapeutic drug monitoring, high performance liquid chromatography, pharmacokinetics

Received: 21 June 2015 / Accepted: 03 August 2015

\section{Background}

Tacrolimus belongs to the macrolid group isolated from Streptomycestzukubaensis. Immunosuppression is achieved by inhibiting calcineurin with the prevention of signal transmission at cell level, necessary for the activation of $\mathrm{T}$ limphocytes [1]. Its efficacy in immunosuppression in patients with kidney transplant as well as in other cases, has been proven by clinical studies [2].

Therapeutic drug monitoring should be an integrated concept in clinical practice, especially in the case of administration of drugs with a low therapeutic index or in the case of administering drugs to a special population group such as patients with chronic kidney disease (CKD) and kidney transplant. On one hand, the purpose of monitoring is personalising the therapeutic regimen and on the other hand improving the treatment [3].

The end stage of CKD implies the initiation of some measures for renal function substitution, and among the existing therapies kidney transplantation is by far one of the elective methods, considering both the costs for the health system as well as the improvement of the patient's condition. [4]
This specific patient population must be permanently administered immunosuppressive therapy in order to maintain the viability of kidney allograft and to reduce chronic or acute rejection phenomena. Taking all this into account, as well as the fact that the studied drug belongs to a drug class with a narrow therapeutical range it is necessary to monitor therapy based on the pharmacokinetic criteria, in view of increasing the efficacy of therapy and to diminish drawbacks generated by underdosage or overdosage [5].

\section{Purpose of research}

Monitoring the pharmacotherapy with tacrolimus is realised in order to improve its efficiency and safety, knowing the vulnerability of patients who are administered such therapy and the problems that occur when the therapeutic range is surpassed, causing the possible onset of toxic phenomena or therapy inefficiency.

The aim of the study was a comparative analysis of the clinical, laboratory and pharmacokinetic parameters in patients with kidney transplant treated with tacrolimus.

\section{Material and method}

A retrospective study was conducted between August $9^{\text {th }}$, 2010 and October 16 ${ }^{\text {th, }} 2014$ on a group of 36 patients, 
clinical and biological data being taken from the medical records of Ambulatory Clinic of The Clinical Institute of Urology and Transplant Cluj Napoca, where the blood concentration of the studied drug was periodically determined, however information was also taken from the documents of the Nephrology Department of the Mures County Clinical Hospital, where the patients are monitored post-transplant.

We included all patients that were under triple immunosuppressive regimen with orally administered tacrolimus, mofetil mycophenolate and prednisone.

The current study has been approved by the Ethics Committee of the County Clinic Hospital of Tirgu-Mures.

The monitoring of the efficacy of therapy (tacrolimus twice daily) was achieved by periodical measurement of tacrolimus level in the blood.

The determination of the blood concentration of tacrolimus was achieved by a validated high-performance liquid chromatography method combined with mass spectrometry. In patients from the studied group, the blood concentration at steady- state was monitored at over 3 months after kidney transplantion, at variable time ranges: 1, 2 or 3 times a year, the blood samples being taken at 12 hours after the last dose, just before the following dose $\left(\mathrm{C}_{\min (\text { trough })}\right.$ - residual concentration).

Our study population was divided in two groups in order to obtain a comparative analysis of the parameters, patients with renal allograft from dead brain donors (group 1) and patients with renal allograft from living donors (group 2). The results are presented as mean $\pm \mathrm{SD}$ (standard deviation). The $T$ student test has been used for unpaired data to compare the mean laboratory results between the two groups and when the data did not conform to the normality test Kolmogorov-Smirnov, Mann-Whitney test has been used. The statistical analysis was done with the use of the statistic programme Graph Pad Prism 6, and p was considered statistically significant under 0.05 .
Table I. Demographic data of patients

\begin{tabular}{lc}
\hline Gender ratio (male/female) $(\mathrm{n})$ & $20 / 16$ \\
Recipient age in years at the admission (mean $\pm \mathrm{SD})$ & $43 \pm 11$ \\
Age in month of renal graft, (mean $\pm \mathrm{SD})$ & $61 \pm 36$ \\
Weight, $\mathrm{kg}$ (mean $\pm \mathrm{SD}$ ) & $73,13 \pm 15,65$ \\
\hline Primary renal disease, $\mathrm{n}(\%)$ & \\
Chronic Glomerular Nephropathy & $15(42)$ \\
Diabetic Nephropathy & $7(19)$ \\
ADPKD & $6(17)$ \\
Congenital Reflux Nephropathy & $3(8)$ \\
Chronic Tubulointerstitial Nephritis & $3(8)$ \\
Nephroangiosclerosis & $1(3)$ \\
Lupus Nephritis & $1(3)$ \\
\hline Type of Transplant, $\mathrm{n}$ (\%) & \\
Dead brain donor (Group 1) & $21(58)$ \\
Living donor (Group 2) & $15(42)$ \\
\hline
\end{tabular}

\section{Results}

A number of 36 patients met the inclusion criteria, mean age of patients was of 43 years $( \pm 11)$ at the moment of inclusion in the study,with a mean age of renal allograft of 61 months( \pm 36$)$. Regarding the patients' gender, male patients were predominant $55 \%(\mathrm{n}=20)$. Analysing the etiology of CKD, pre-transplant most patients were diagnosed with chronic glomerular nephropathy $53 \%(\mathrm{n}=19)$, autosomal dominant polycystic kidney disease (ADPKD) $17 \%(\mathrm{n}=6)$, diabetic nephropathy $8 \%(\mathrm{n}=3)$, congenital reflux nephropathy $8 \%(\mathrm{n}=3)$, nephroangiosclerosis $3 \%$ $(\mathrm{n}=1)$, lupus nephritis $3 \%(\mathrm{n}=1)$. Clasifying patients after the type of renal allograft, we have noticed that the patients who received an allograft from a dead brain donor are predominant $58 \%(\mathrm{n}=21)$. [Table I]

Comparative analysis between the two groups shows that serum creatinine was significantly higher in dead brain donors [Table II]. Blood glucose was significantly higher in the same group but this is not clinically relevant considering the fact that 6 patients of group 1 had in CKD etiology diabetic nephropathy [Tabel I, II].

Table II. Laboratory parameters and comparisons between groups of patients

\begin{tabular}{|c|c|c|c|c|c|}
\hline \multirow[t]{2}{*}{ Parameter } & \multicolumn{2}{|c|}{$\begin{array}{l}\text { Dead brain donor } \\
\text { Group } 1(n=21)\end{array}$} & \multicolumn{2}{|c|}{$\begin{array}{l}\text { Living Donor } \\
\text { Group } 2(n=15)\end{array}$} & \multirow[t]{2}{*}{$P$} \\
\hline & mean $\pm S D$ & Median"(Min-Max) & mean $\pm S D$ & Median (Min-Max) & \\
\hline ALT (UI/L) & $25,3 \pm 23,8$ & $17(6-211)$ & $19,2 \pm 7,7$ & $18(8-44)$ & $\mathrm{NSa}$ \\
\hline AST (UI/L) & $21 \pm 10,5$ & $18(9-90)$ & $18,9 \pm 4,2$ & $18,5(12-29)$ & $\mathrm{NSa}$ \\
\hline Total bilirubin (mg/dL) & $0,52 \pm 0,22$ & $0,47(0,13-1,21)$ & $0,62 \pm 0,39$ & $0,5(0,21-2,05)$ & $\mathrm{NSa}$ \\
\hline Serum protein $(\mathrm{g} / \mathrm{dL})$ & $6,95 \pm 0,46$ & $6,9(5,4-8)$ & $7,02 \pm 0,35$ & $7(6,2-7,7)$ & $\mathrm{NSb}$ \\
\hline Cholesterol (mg/dL) & $192,8 \pm 43,0$ & 192(107-338) & $203,3 \pm 40,1$ & 201(124-302) & $\mathrm{NSb}$ \\
\hline Triglyceride (mg/dL) & $138,8 \pm 74,2$ & $119(49-454)$ & $134,6 \pm 78,5$ & $113(41-462)$ & NSa \\
\hline VLDL (mg/dL) & $27,8 \pm 14,8$ & $24(10-91)$ & $27,2 \pm 16,2$ & 23(8-93) & NSa \\
\hline Serum Creatinine (mg/dL) & $1,31 \pm 0,49$ & $1,22(0,51-3,64)$ & $1,10 \pm 0,34$ & $1,1(0,62-2,27)$ & $0,002 a$ \\
\hline Uric Acid (mg/dL) & $5,99 \pm 1,12$ & $5,9(4-8,6)$ & $5,59 \pm 1,40$ & $5,8(2,5-7,5)$ & $\mathrm{NSb}$ \\
\hline Potassium (mmol/L) & $4,36 \pm 0,45$ & $4,3(3,5-5,6)$ & $4,26 \pm 0,39$ & $4,2(3,5-5,1)$ & $\mathrm{NSa}$ \\
\hline Glucose (mg/dL) & $104,2 \pm 40,6$ & $94(61-345)$ & $86,3 \pm 10,1$ & $84(66-111)$ & $0,0001 a$ \\
\hline 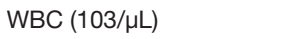 & $7,00 \pm 2,94$ & $6,24(2,45-22,09)$ & $7,21 \pm 2,06$ & $6,95(3,68-11,92)$ & $\mathrm{NSa}$ \\
\hline RBC $(106 / \mu \mathrm{L})$ & $4,61 \pm 0,78$ & $4,69(2,08-6,48)$ & $4,71 \pm 0,47$ & $4,75(3,63-5,83)$ & $\mathrm{NSa}$ \\
\hline Haemoglobin (g/dL) & $13,38 \pm 2,43$ & $13,3(7,9-18,3)$ & $13,49 \pm 1,38$ & $13,8(9,7-17,3)$ & NSa \\
\hline Hematocrit (\%) & $41,73 \pm 6,94$ & $41(25,8-56,6)$ & $41,80 \pm 3,54$ & $42(32,3-48,9)$ & NSa \\
\hline Platelets $(103 / \mu \mathrm{L})$ & $215,6 \pm 47,7$ & 206(99-372) & $233,4 \pm 62,7$ & 238(106-423) & $\mathrm{NSa}$ \\
\hline
\end{tabular}


During monitoring 252 blood determinations of tacrolimus concentration at steady-state $\mathrm{C}_{\min (\text { trough) }}$ have been performed with a mean of 7 determinations per patient. 58 of these determinations were not in the therapeutic range (4-11 ng/ml), established by the current therapeutic guides [6]. 44 samples pointed at underdose, being under $4 \mathrm{ng} / \mathrm{ml}$ and 14 determinations were over $11 \mathrm{ng} / \mathrm{ml}$, being over the therapeutic range, pointing at overdosage and toxic level. The level of tacrolimus varied in a rather wide range, the smallest value being of $1,1 \mathrm{ng} / \mathrm{ml}$, and the highest value being of $29,7 \mathrm{ng} / \mathrm{ml}$. [Table III]

The daily dose of tacrolimus administered is slightly higher at patients from studied group 1, and GFR mean (Glomerular Filtration Rate) is lower, raising the risk of surpassing the therapeutic range due to the low renal clearance. For GFR we used the simplified form of the 4-variable MDRD (Modification of Diet in Renal Disease) Study Equation: eGFR $=186 \mathrm{x}$ (Serum Creatinine) ${ }^{-1.154} \mathrm{x}$ (Age) $)^{-0.203} \times(0.742$ if the subject is female) $\mathrm{x}(1.210$ if the subject is black) [7]. Therefore, the level of overdosage at patients from the studied group 1 is higher comparatively with that of patients from the studied group 2. The level of underdosage is also high at these patients. Regarding the post-transplant complications, infections were frequent (bacterial, fungal, viral), urological complications, chronic allograft dysfunctions, anemia, neoplasias secondary to immunosuppression. [Table III]

\section{Discussions}

We can state that kidney transplant represents a real advantage regardless of the type of donors for patients with end stage CKD by raising life expectancy as well as their quality of life. Nevertheless, it seems that even if the technical procedures are followed regarding the organ prelevation, from a clinical perspective the renal allografts from living donors are more viable. $[8,9]$. The economic benefit, relevant for efficient health systems, is added to the clinical advantage especially if it involves preemptive treatment, which on one hand relieves the health system of the costs that are required by the current dyalisis methods and on the other hand the number of comorbidities, which might appear following the use of previously mentioned methods of renal substitution, are significantly reduced [10].

The comparison of the laboratory parameters of the patients divided in the two groups according to the renal al- lograft: from dead brain donor (Group 1) and from living donor (Group 2), is justified in the specific case of the current study first of all from the point of view of the patients' clinical development, the preservation of the renal allograft being superior from living donors [8].

The mean level of plasma creatinine at group 1 is higher, in agreement with other studies [11], and GFR is automatically lower, $p=0,005$ at these patients, therefore an increase of drug concentration in the blood is noticed at this group of patients for whom renal clearance is impaired. One of the reasons for increased dose per day (Table III) at these category of patients, statistically significant $(p=0,003)$, is caused by the difficulty of maintainig the drug level in the therapeutic range. Moreover, we cannot overlook the lower renal clearance in this group of patients, which added to larger doses can cause nephrotoxic phenomena following overdose [12].

No cases of iatrogenic diabetes secondary to therapy with tacrolimus and prednisone were noticed at the studied groups. What should be considered is the fact that in the case of patients with diabetes prior transplant, metabolic alterations are likely to occur secondary to tacrolimus and prednisone[13].

There are a number of factors which can influence the pharmacokinetics of tacrolimus, many of these being found in patients included in this study. Among these we should mention first of all, the simultaneous immunosuppressive medication such as mycophenolate mofetil, where research shows increased serum levels of tacrolimus [14]. Corticotherapy may also represent a significant underlying factor, since it uses the same isoenzymes of cytochrome $\mathrm{P}_{450}$ for byotransformation (CYP3A4), leading to a decrease of tacrolimus level in the blood, having a slight enzymatic inductor effect [15]. Another important element is the age of renal allograft, the patients age at the moment of transplant, the medication administered for other comorbidities such as calcium-channel blockers (felodipine) for the treatment of hypertension -4 patients from group 1 and 2 patients from group 2 [16]; theophylline for the treatment of asthma - 1 patient from group 1 [17], ketoconazole, inhibitor of CYP3A4, for the treatment of mycotic infections - 2 patients from group 1 and 1 patient from group 2 [18],the patients' diet (grapefruit juice, intake of pomelo, foods that may act as enzymatic inhibitors of CYP3A4) [19,20], CYP3A4 polymorphism [21]. All of the above

Table III. Pharmacokinetic characteristics of the groups

\begin{tabular}{|c|c|c|c|c|c|c|c|c|c|c|c|c|}
\hline \multirow{2}{*}{ 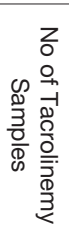 } & \multirow{2}{*}{ 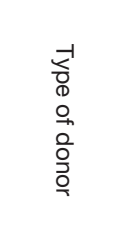 } & \multirow{2}{*}{ 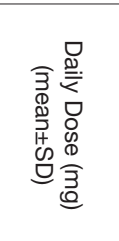 } & \multirow{2}{*}{ 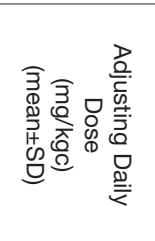 } & \multirow{2}{*}{ 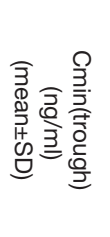 } & \multirow{2}{*}{ 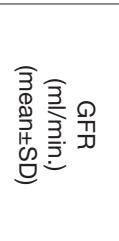 } & \multirow{2}{*}{ 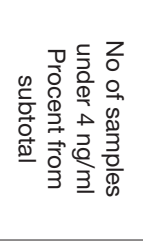 } & \multirow{2}{*}{ 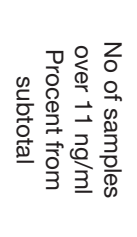 } & \multicolumn{5}{|c|}{ Posttransplant complications } \\
\hline & & & & & & & & 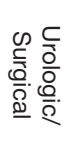 & $\begin{array}{l}\overline{\bar{\sigma}} \\
\stackrel{0}{0} \\
\stackrel{0}{\overline{0}} \\
\overline{5}\end{array}$ & $\begin{array}{l}\frac{7}{3} \\
\frac{1}{3} \\
\frac{1}{\omega}\end{array}$ & 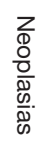 & 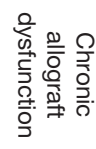 \\
\hline 65 & Living & $2,84 \pm 0,9$ & $0,04 \pm 0,019$ & $5,94 \pm 2,7$ & $66 \pm 17$ & $11(17 \%)$ & $1(2 \%)$ & 0 & 9 & 0 & 1 & 1 \\
\hline \multirow[t]{2}{*}{187} & Dead brain & $3,42 \pm 1,4$ & $0,049 \pm 0,019$ & $6,48 \pm 3,7$ & $59 \pm 21$ & $33(18 \%)$ & $13(7 \%)$ & 5 & 12 & 4 & 1 & 3 \\
\hline & & ${ }^{*} \mathrm{P}=0,003$ & ${ }^{*} \mathrm{NS}$ & ${ }^{*} N S$ & ${ }^{*} \mathrm{P}=0,005$ & & & & & & & \\
\hline
\end{tabular}


may cause interactions when associated, raising thus the serum level of tacrolimus.

\section{Conclusions}

Kidney transplant is one of the efficient methods of treatment for the end stage of CKD. That is why it is recommended to change the population's perception toward organ donation, in case of dead brain patients as well as for living patients, namely in case of altruistic donations.

Consequently we must admit that the interindividual variability (CYP3A4 polymorphism)should be taken into account especially in the case of immunosuppressive therapy, at patients with kidney transplant. The improvement of immunosuppressive therapy cannot be achieved only through a more precise adjustement, according to the clearance of creatinine, body weight, through the change of the interval of administering dosage in order to maintain tacrolimus level in the therapeutic window. In the same time, a clinical and paraclinical assessment at shorter intervals especially, maybe every two months, in the case of patients who received the renal allograft from a dead brain donor is rendered mandatory. In this way the monitoring of post transplant complications is more rigurously controlled facillitating early therapeutic intervention.

Regarding the tailoring of dose, there are two options available: either dose adjustment, which is the current practice, or the adjustment of the time interval between administrations, especially at patients of group 1 who are more vulnerable avoiding thus underdosage and inefficient therapy or overdosage followed by toxic phenomena. Continuous monitoring based on the pharmacokinetic criteria is highly recommended in case of a special group of patients such as that of patients with CKD and kidney transplant, since dose administration is still done empirically. Therefore, tailoring the dose must be done according to the level of the blood concentration of the immunosuppressant drug, without overlooking other cofactors described in this study, which might interfere and alter an optimum level of therapeutical concentration.

Taking into account the conclusions that we have reached as a result of our research as well as the limitations of this study, represented by the small number of transplanted patients included in this study (those belonging to Mures area) and on the other hand, the data collected from only one transplant centre, we aim at conducting a further study, which might comprise a larger group of patients, from more counties, possibly patients from more transplant centers from our country for a comparative analysis. In addition a study of populational pharmacokinetics could be conducted, taking into account the characteristics of this special population with CKD and kidney transplant.

\section{Acknowledgements}

This paper was published under the frame of European Social Found, Human Resources Development Operational Programme 2007-2013, project no. POSDRU/159/1.5/S/136893.

\section{References}

1. Venkataraman L, Burakoff SJ, Sen R. FK-506 inhibits antigen receptormediated induction of C-rel in B and T lymphoid cells. J Exp Med. 1995;181:1091-1099.

2. Plosker GL, Foster RH. Tacrolimus: a further update of its pharmacology and therapeutic use in the management of organ transplantation. Drugs. 2000;59:323-389.

3. Kang JS, Lee MH. Overview of therapeutic drug monitoring. The Korean Journal of Internal Medicine. 2009;24:1-10.

4. Wong G, Howard K, Chapman JR, Chadban S et al. Comparative survival and economic benefits of deceased donor kidney transplantation and dialysis in people with varying ages and co-morbidities. PLoS ONE 2012;7:1-9.

5. Schiff J, Cole E, Cantarovich M. Therapeutic monitoring of calcineurin inhibitors for the nephrologist. Clin J Am Soc Nephrol. 2007;2:374-384.

6. Wallemacq P, Armstrong WW, Brunet $M$ et al. Opportunities to optimize tacrolimus therapy in solid organ transplantation: report of the european consensus conference. Ther Drug Monit. 2009;31:139-152.

7. Stevens LA, Coresh J, Greene T, Levey AS. Assessing kidney functionmeasured and estimated glomerular filtration rate. N Engl J Med. 2006, 354:2473-2483.

8. Koo DDH, Welsh KI, McLaren AJ et al. Cadaver versus living donor kidneys: Impact of donor factors on antigen induction before transplant. Kidney International 1999;56:1551-1559.

9. Gjertson DW, Cecka JM. Living unrelated donor kidney transplantation. Kidney International 2000;58:491-499.

10. Kanellis J. Justification for living donor kidney transplantation. Nephrology 2010;15:S72-S79.

11. Zivcic-Cosic S,Trobonjaca Z, Racki S. Immunosuppressive treatment for kidney transplantation. Prilozi 2011;32:87-103.

12. Naesens M, Kuypers DRJ, Sarwal M. Calcineurin inhibitor nephrotoxicity. Clin J Am Soc Nephrol 2009;4:481-508.

13. Michael F. Crutchlow, Roy D. Bloom. Transplant- associated hyperglycemia: A new look at an old problem.Clin J Am Soc Nephrol 2007;2:343-355.

14. Pirsch J, Bekersky I, Vincenti F et al. Coadministration of tacrolimus and mycophenolate mofetil in stable kidney transplant patients: pharmacokinetics and tolerability. J Clin Pharmacol 2000;40:527-532.

15. Hasselink DA, Ngyuen $\mathrm{H}$, Wabbijin M et al. Tacrolimus dose requirement in renal transplant recipients is significantly higher when use in combination with corticosteroids. Br J Clin Pharmacol 2003;56:327-330.

16. Butani L, Berg G, Makker SP. Effect of felodipine on tacrolimus in a renal transplant recipient. Transplantation 2002;73:159.

17. Boubenider S, Vincent I, Lambotte $\mathrm{O}$ et al. Interaction between theophylline and tacrolimus in a renal transplant pacient. Nephrol Dial Transplant 2000;15:1066-1068.

18. Floren LC, Bekersky I, Benet $L Z$ et al. Tacrolimus oral bioavailability doubles with coadministration of ketoconazole. Clin Farmacol Ther 1997;62:41-49.

19. Michelangelo V, Piero D, Elisa C et al. Grapefruit juice and kinetics of tacrolimus. J Am Soc Nephrol 2001;12:862.

20. Egashira K, Fukuda E, Onga T et al. Pomelo-induced increase in the blood level of tacrolimus in a renal transplant pacient. Transplantation 2003;75:1057.

21. Kurzawski M, Dąbrowska J, Dziewanowski Ket al. CYP3A5 and CYP3A4, but not $\mathrm{ABCB} 1$ polymorphisms affect tacrolimus dose-adjusted trough concentrations in kidney transplant recipients. Pharmacogenomics 2014;15:179-188. 